\title{
Dynamic exponent in Extremal models of pinning.
}

\author{
Supriya Krishnamurthy ${ }^{1}$, Anne Tanguy ${ }^{2}$ and Stéphane Roux ${ }^{3}$ \\ 1: Laboratoire de Physique et de Mécanique des Milieux Hétérogènes, \\ Ecole Supérieure de Physique et Chimie Industrielles de Paris, \\ 10, rue Vauquelin, 75231 Paris Cedex 05, France. \\ 2: Université de Lyon I, 43 Bd. du 11 Novembre 1918, \\ 69622 Villeurbanne, France. \\ 3: Laboratoire Surface du Verre et Interfaces, \\ Unité Mixte de Recherche CNRS/Saint-Gobain, \\ 39 Quai Lucien Lefranc, BP 135, F-93303 Aubervilliers Cedex, France.
}

(April 18, 2017)

\begin{abstract}
The depinning transition of a front moving in a time-independent random potential is studied. The temporal development of the overall roughness $w(L, t)$ of an initially flat front, $w(t) \propto t^{\beta}$, is the classical means to have access to the dynamic exponent. However, in the case of front propagation in quenched disorder via extremal dynamics, we show that the initial increase in front roughness implies an extra dependence over the system size which comes from the fact that the activity is essentially localized in a narrow region of space. We propose an analytic expression for the $\beta$ exponent and confirm this for different models (crack front propagation, Edwards-Wilkinson model in a quenched noise, ...).
\end{abstract}

The propagation of a front in a noisy environment has been the subject of active research in the past. In particular, after Family and Vicsek proposed a scaling form for the evolution of the roughness of fronts and surfaces from Langevin equations, numerous analytical and numerical works have verified these laws in a wide variety of models. Various reviews cover this rich field [1] 3].

More recently, the quenched (i.e. time-independent) nature of the noise was recognised as playing a significant role in front propagation. Unfortunately, in spite of a few key works, analytic modelling of such a depinning transition is rather scarce: Dynamic Renormalization Group studies have been proposed for the Edwards-Wilkinson model with quenched disorder [4 6 however in $1+1$ dimensions some of the predicted exponents are quite far from their estimate obtained in numerical simulations. It has been proposed that the Kardar-Parisi-Zhang model in a quenched environment can be described by a directed percolation related model, mostly on the basis of numerical agreement between measured exponents [7,8].

Such models are relevant for a number of pinning phenomena such as crack propagation [9 13, wetting phenomena [14,15], vortex pinning in type-II superconductors [16] and solid friction 117, 18].

Growth models in $1+1$ dimensions are often studied through the evolution in time $\theta$, (defined as the number of growth steps $t$ divided by the system size $L$ ), of the roughness, $w(\theta)$, of an initially flat front $w(0)=0$. The roughness — standard deviation of the front — in a system of size $L$ obeys the scaling form

$$
w(\theta)=\theta^{\beta} \varphi\left(\frac{L}{\theta^{1 / z_{1}}}\right)
$$

where

$$
\varphi(x)= \begin{cases}\text { cst } & \text { for } x \ll 1 \\ x^{\zeta} & \text { for } x \gg 1\end{cases}
$$

and $\zeta=\beta z_{1}$. The exponent $z_{1}$ is referred to as the dynamic exponent since it relates space and time, whereas $\zeta$ describes the roughness of the front (Hurst or roughness exponent), i.e. the scaling of the pair correlation function for equal-time positions along front. In most cases of annealed noise, there is no need [19] to introduce any other exponents, since at late stages, when the overall roughness has reached the saturation value, the full two point correlation function of the front at different locations and times reveals a similar scaling between space and time: $\Delta x \propto \Delta \theta^{1 / z_{1}}$.

The aim of this letter is to show that for a class of quenched disorder depinning models, the early time development of the roughness does not obey such a law. Rather, it implies an extra $L$ dependence in the expression of $w$, from which $\zeta=\beta z_{1}$ is violated.

We focus more specifically on a class of models introduced by Tanguy et al [21] obeying extremal dynamics. The front is defined by its position $z=h(x, t)$. An external driving $F$ allows to exert a pressure on the front which is 
however biased by the position of the front, so that at each site $x$, the force is $f(x, t)=F(G \star h)$ where the $\star$ denotes a convolution product, and $G$ is a function which is specific to the physical problem studied. The local part of $G$ is adjusted so that $G(0)=-\sum_{x \neq 0} G(x)$. In order to normalize this kernel, we choose as a convention $G(0)=-1$. The key feature is that $G$ may have power-law tails which determine the universality class of the problem. For a planar crack, Gao and Rice [13] have shown that (to first order in $h$ ), $G$ decays as $r^{-2}$. The same holds for the motion of the triple line of a liquid-glass interface intersecting a solid surface, in a wetting problem with a semi-infinite liquid surface in weak gravity. If the liquid/gas system is confined between two parallel plates the $G$ function decays more abruptly, as $r^{-3}$. In the mean-field limit, the $G$ function does not depend on distance. For the sake of convenience, this model has thus been generalized to any power-law form for $G$ :

$$
G(r) \propto r^{-\alpha}
$$

In our simulations, periodic boundary conditions are implemented and thus we adjust $G$ to match such a periodicity: namely $G(r) \propto \sin (\pi r / L)^{-\alpha}$. Such an expression is exact for $\alpha=2$, and is a convenient means to implement the periodic b.c. in other cases.

The environment is represented by quenched heterogeneities, $\eta(x, z)$, which can block the front whenever $f(x, t)<$ $\eta(x, h(x, t))$. The $\eta$ values are considered to be uncorrelated, positive numbers, randomly picked from a uniform distribution over the interval $[0 ; 1]$. Thus for $F=0$, the interface does not move. At each time step, the force is increased slowly from 0 up to the level where one site $x^{*}$ (the active site) can depin. This site jumps to the next obstacle $\eta$ at a random distance along the $z$ direction. This distance is chosen again from a uniform distribution over the interval $[0 ; d]$. The external loading is immediately brought back to zero so that no other sites can move simultaneously. The same step is repeated indefinitely. $d$ is a free parameter. It has been checked that $d$ plays no role in the statistical properties of the model in the steady state.

The steady state properties of this model have been studied in detail numerically 20 22]. It has been shown in particular that for $\alpha \leq 1$, the model is in the mean field regime, as can be easily inferred from the $\alpha=0$ case. No spatial structure appears, and the front is an uncorrelated white noise. For $\alpha \geq 3$, the long-range kernel is dominated by the short wavelength cut-off, and is thus equivalent to the case where $G$ is the second derivative of a Dirac distribution, i.e. the local force is proportional to the external loading and the local curvature of the front. This is the Edwards-Wilkinson or "Laplacian" case with quenched disorder. The front has a "super-rough" structure with a roughness exponent $\zeta \approx 1.2$ [23]. The steady state properties of this dynamics is fairly rich and a number of scaling properties can be observed for the front structure, the time evolution of the activity, and of the driving force necessary to depin the interface. In the intermediate range $1<\alpha<3$, similar properties are observed with scaling exponents which vary continuously with $\alpha$.

One especially interesting property can be studied in order to characterize the spreading of activity in space and time: Let $x^{*}\left(t_{0}\right)$ be the active site at time $t_{0}$, and $x^{*}\left(t_{0}+\Delta t\right)$ at time $t_{0}+\Delta t$. Note that here we use as a time the total number of moves $t$ rather than the number of moves per site $\theta=t / L$. We study the statistical distribution $p(\Delta x, \Delta t)$ of $\Delta x=\left|x^{*}\left(t_{0}+\Delta t\right)-x^{*}\left(t_{0}\right)\right|$ for a fixed $\Delta t$. It obeys the scaling form

$$
p(\Delta x, \Delta t)=\Delta t^{-1 / z_{2}} \Psi\left(\frac{\Delta x}{\Delta t^{1 / z_{2}}}\right)
$$

where

$$
\Psi(x)= \begin{cases}c s t & \text { for } x \ll 1 \\ x^{-b} & \text { for } x \gg 1\end{cases}
$$

where $b$ is equal to $\alpha$. Such a scaling form was first introduced by Furuberg et al studying invasion percolation [24]. Thus, in the steady state, we see that the activity spreads typically over distances $\Delta x \propto \Delta t^{1 / z_{2}}$. In this sense, $z_{2}$ is actually the "dynamic exponent", and it indeed governs all correspondences between time and space in the steady state. Note however that a different convention is now used for $z$, because of the definition of time. In particular the activity has spread over the entire system for a time equal to $t \propto L^{z_{2}}$, hence $\theta=t / L \propto L^{z_{2}-1}$. Thus actually $z_{2}$ should be compared to $z_{1}+1$. In models with extremal dynamics, $z_{2}$ can easily be related to the roughness exponent of the front [25]21]. After a time $\Delta t$, the activity remains localized in a region of extend $\Delta x$. Over this region, the front moves by a typical distance of order $\Delta z \propto \Delta x^{\zeta}$. Thus the number of time steps required to travel by this amount scales as $\Delta x \Delta z \propto \Delta x^{1+\zeta}$, hence

$$
z_{2}=1+\zeta
$$


This simple argument has been checked to be obeyed precisely in numerical simulations for $1<\alpha<3$. Let us however note that it breaks down in the Laplacian case $\alpha \rightarrow \infty$ where $z_{2} \approx 2.0$ and $\zeta \approx 1.25$. However, the situation when $\zeta>1$ is known to display some pathological behaviors.

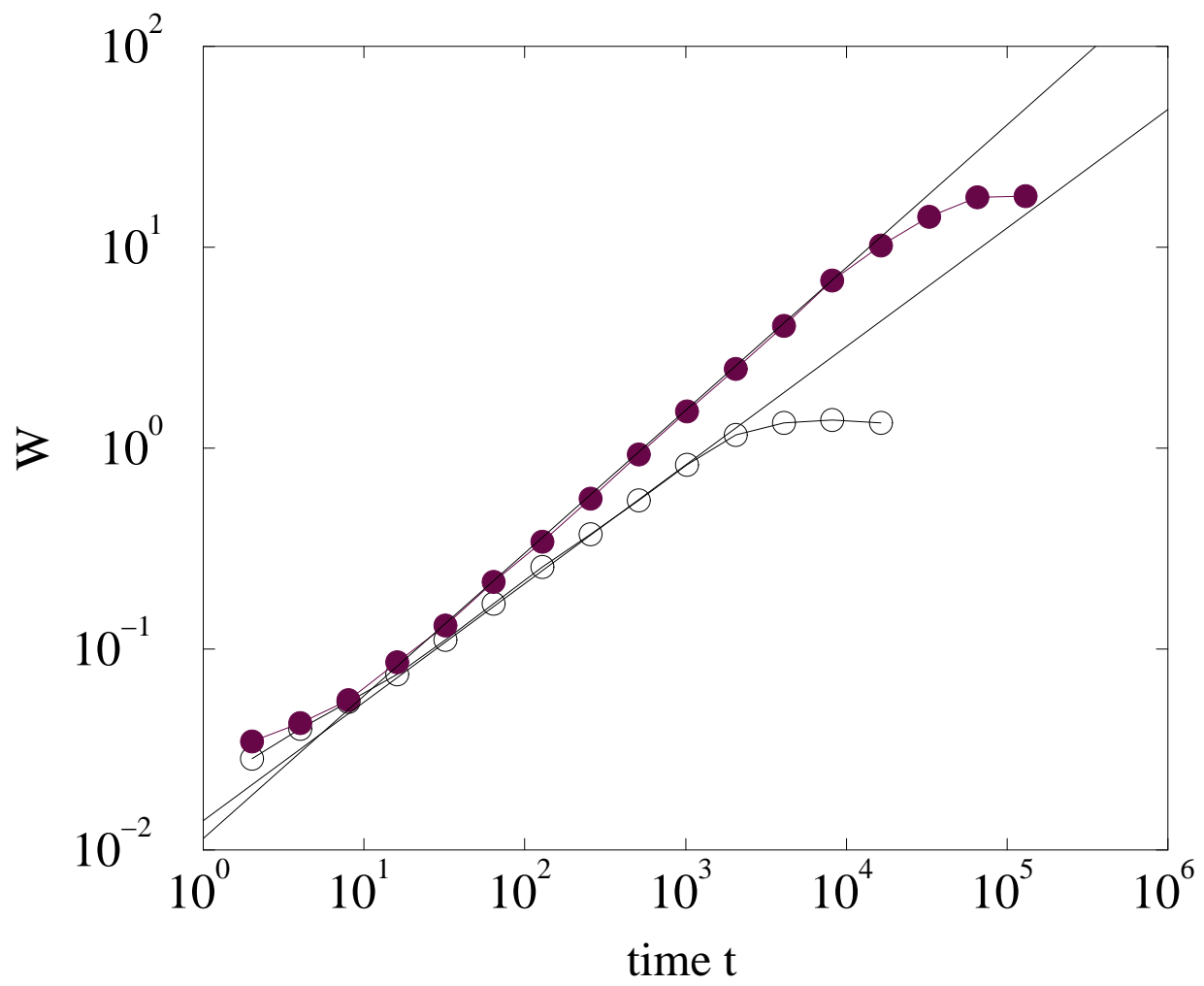

FIG. 1. Log-log plot of the overall roughness of the interface as a function of time for two values of the $\alpha$ parameter $\alpha=2$ (symbol $\circ$ ) and $\alpha=3$ (symbol $\bullet$ ). The best power-law fits are shown as plain lines. The values of the slopes are reported in Table 1 The size of the system is $\mathrm{L}=1024$.

We now consider the early stages of the front growth. We assign an initial flat configuration for the front $z=0$ and study the evolution of the front roughness, in order to characterize $z_{1}$ through the scaling relation Eq. 1. Figure 1 shows a log-log plot of the overall roughness for the crack model, $\alpha=2$, and for $\alpha=3$. In both cases, we indeed measure a power-law, $w \propto t^{\beta}$, with $\beta \approx 0.61$ and 0.70 respectively. Table If gives the different values of $\zeta$ and $z_{2}$ from Ref. [21], and $\beta$ from the numerical simulations of the present study. We note that if we blindly apply the relation $z_{1}=\zeta / \beta$, we find a severe discrepancy with $z_{2}-1$ which cannot be attributed to numerical uncertainties. For instance, if $\alpha=2, \zeta / \beta=0.58$, and $z_{2}-1=0.35$. Let us note that this observation invalidates the numerical determination of the $z$ exponent published for example in Refs. [4,5] (moreover, in these articles the analytical expression proposed for $z$, using RG analysis, refers to another driving mode that could be interesting to compare with extremal models). This is the main message of this paper: The standard relation $z=\zeta / \beta$ breaks down for quenched disorder growth models with extremal dynamics. 


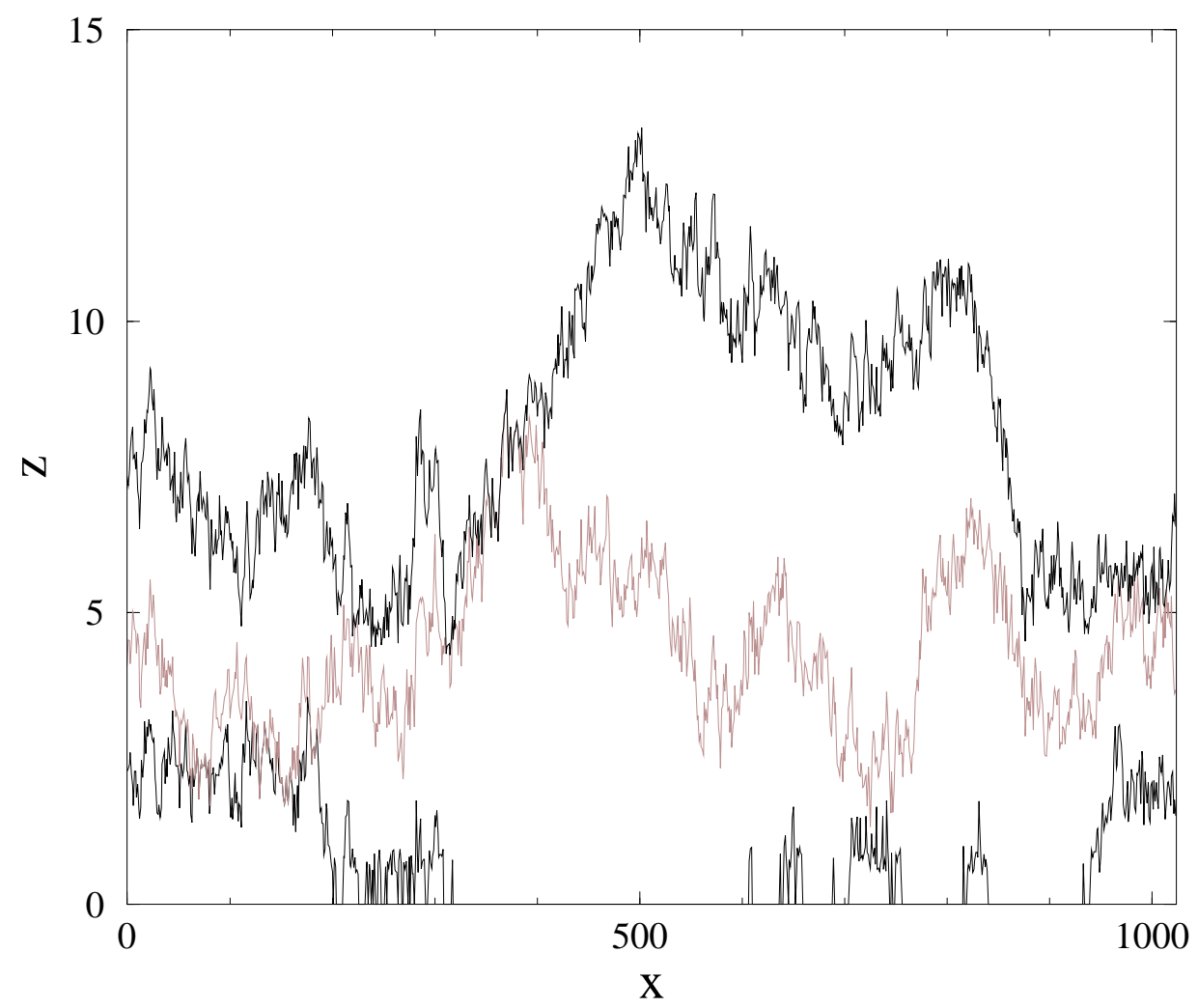

FIG. 2. Shape of the first stages $t=1000,6000$ and 11000 of the interface for $\alpha=2$ and $L=1024$. We note that the activity is not equally distributed along the interface, but rather only a localized part grows and reaches a steady state conformation before growing and invading the flat region. This observation expressed in quantitative terms gives our prediction of $\beta$.

We can go one step beyond the numerical study reported so far and estimate the value of $z_{1}$ from the steady state roughness exponent. To this end, it is informative to look at the shape of the front in the initial regime. Figure 2 shows such an example for $\alpha=2$ and $L=1024$. We observe that the front remains pinned along its initial flat geometry for a large time, and thus the interface moves only in a confined region of space. This region progressively grows, and invades the flat part, but still continues its propagation along the $z$ direction. This is in marked contrast with annealed noise type growth where the activity is delocalized along the entire front. Moreover, as can be noted from Figure 1, the cross-over to the saturation regime is quite steep. This suggests that the depinned parts of the front have already reached their steady state roughness.

Let us now translate this argument into quantitative terms. We assume that the interface is depinned along one single interval of length $\ell$. Along this interval, the roughness assumes its asymptotic value, i.e. the typical height will be of order $h \approx \ell^{\zeta}$. Therefore the time needed to reach this position is

$$
t \propto \ell^{1+\zeta}
$$

Generally, as one follows the time development of the roughness, one introduces as a time the number of moves per site, $\theta=t / L$. Ignoring subdominant terms coming from the average height of the interface, we compute the overall roughness, $w$, as

$$
w^{2} \propto \frac{\ell^{2 \zeta+1}}{L}
$$

where the multiplicative $\ell / L$ term comes from the weight of the depinned part of the interface as compared to the rest. Therefore the $\beta$ exponent is readily estimated from the elimination of $\ell$ in the two above equations, and thus

$$
w \propto \theta^{\beta} L^{\beta-1 / 2}
$$

with

$$
\beta=\frac{\zeta+1 / 2}{\zeta+1}
$$


We note however that there is an extra term depending on $L$ in the rate of growth of the roughness. Thus one cannot use the standard relation $z_{1}=\zeta / \beta$. Instead, we write

$$
w=\theta^{\beta} L^{\beta-1 / 2} \varphi\left(\frac{L}{\theta^{1 / z_{1}}}\right)
$$

The dynamic exponent $z_{1}$ is then found by imposing that in the steady state $w$ is $t$-independent, and $w \propto L^{\zeta}$. This gives the power-law behavior of $\varphi$ for small arguments, $\varphi(x) \propto x^{\zeta+1 / 2-\beta}$, and thus

$$
\beta-\frac{\zeta+1 / 2-\beta}{z_{1}}=0
$$

or using our above expression for $\beta$

$$
z_{1}=\frac{\zeta+1 / 2-\beta}{\beta}=\zeta
$$

Thus the total number of moves necessary to reach the steady state scales as $t=\theta L \propto L^{1+\zeta}$, and thus we recover our previous expression for $z_{2}=1+z_{1}$ where the difference of one simply comes from the definition of time.

\begin{tabular}{|c|c|c|c|c|}
\hline$\alpha$ & $\begin{array}{c}\zeta \\
\text { from Ref. [21] }\end{array}$ & $\begin{array}{c}z_{2} \\
\text { from Ref. [21] }\end{array}$ & $\begin{array}{c}\beta \\
\text { (measured) }\end{array}$ & $\begin{array}{c}\beta \\
\text { from Eq. (10) }\end{array}$ \\
\hline \hline 1.5 & 0.05 & 1.05 & 0.49 & 0.52 \\
2.0 & 0.35 & 1.35 & 0.61 & 0.63 \\
2.5 & 0.65 & 1.65 & 0.65 & 0.70 \\
3.0 & 1.0 & 2.0 & 0.70 & 0.75 \\
$\infty$ & 1.25 & 2.0 & $0.8^{*}$ & 0.78 \\
\hline
\end{tabular}

TABLE I. Values of the exponents $\zeta$ and $z_{2}$ taken from Ref.[21], measured value of $\beta$ from the present work, and predicted value from Eq. (10). ${ }^{*}$ refers to Ref.[23]. 
In Table II, we have reported the value of the $\beta$ exponent estimated from numerical simulation data, and the one obtained from Eq. 10. We note an excellent numerical agreement for all values of $\alpha$ studied.

More generally if other moments of the interface height are computed, a different scaling is expected. Indeed for the moment of order $m$, a similar computation gives $\left\langle(h-\langle h\rangle)^{m}\right\rangle^{1 / m} \propto t^{\beta_{m}}$ with

$$
\beta_{m}=\frac{\zeta+1 / m}{\zeta+1}
$$

Hence, in contrast with annealed models, different moments give rise to different estimates of the $\beta$ exponent. This again underlines the fact that care has to be taken with the interpretation of the latter exponent.

We mentioned that the part of the interface which has moved had already a conformation representative of the steady state regime. In order to check this, we can perform the following test. After the interface has reached the steady state, we choose an arbitrary time $t_{0}$ and record the position of the interface $h_{0}(x)=h\left(x, t_{0}\right)$. Then as the simulation continues, we study the incremental motion of the front, $\Delta h(x, t)=h(x, t)-h_{0}(x)$. The time evolution of the roughness of $\Delta h$ follows indeed the same law as the early stage $t^{\beta}$. This shows that the initial flat configuration behaves as any late stage configuration.

The final question to answer is why the activity is localized during the early stages of front propagation. As a site moves, a large part of the forces it carries is transferred to the nearest neighbours, while a smaller part is transferred to the second neighbours, and so on. The amount of load transfer depends on the distance to the active site as dictated by the kernel $G$ of the model, and hence it depends on $\alpha$. For $\alpha \rightarrow \infty$, the load transfer is local, and only the nearest neighbors are influenced. The amplitude of the change in force is by the definition of the model dependent on the distance the active site advances, and thus it depends on the parameter $d$. For large $d$, one may easily understand that the activity has a tendency to move uniformly in the transverse direction. The first active site will jump by a distance proportional to $d$, and hence its closest neighbours will be pushed forward by such an amplitude that the amplitude of the threshold strength may be insufficient to keep them pinned. In contrast, for small $d$, the threshold distribution may win over the force modification induced by the roughening of the front. As the amplitude of $d$ is reduced, indeed, the activity map shows that the very initial stage is spread over the entire interface. In the very early stages, one measures a roughness exponent $\beta \approx 0.5$ as can be expected from the trivial observation that only a number $t$ of sites move by a single step proportional to $d$, thus $w \propto d t^{1 / 2} L^{-1 / 2}$. After this initial transient, a higher slope takes over. Figure 33 indicates the evolution of $w(t)$ for three values of $d, 1.0,0.1$ and 0.01 . Fitting the time region where on average there has been more than one move per site, $t>L$, gives consistent estimates of $\beta$ as mentioned in Table $\mathbb{E}$. We thus conclude that the above description of the early stage growth is the generic case, which is encountered for any specific choice of the parameter $d$. 


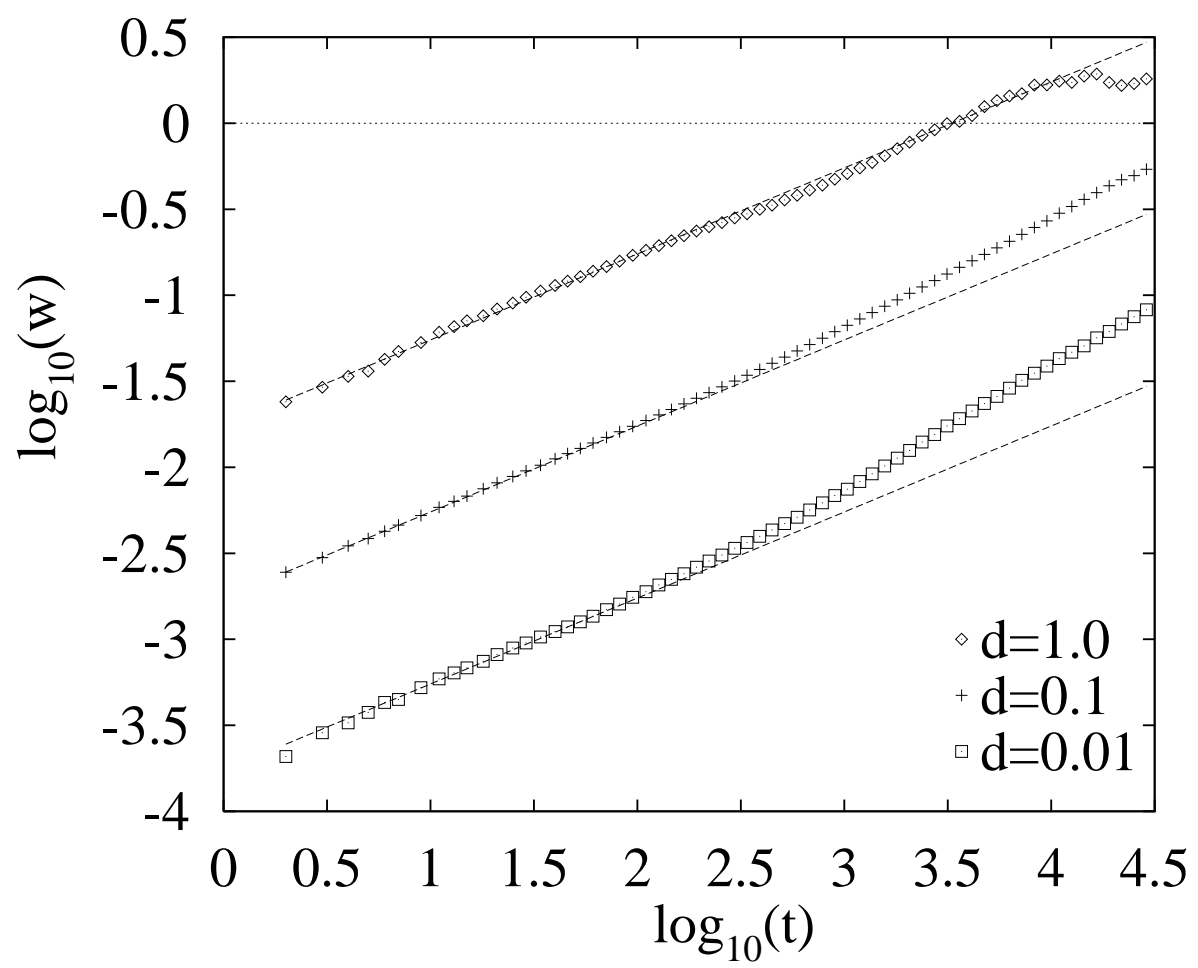

FIG. 3. Log-log plot of the overall roughness versus time for $\alpha=2$ and $L=1024$. Three values of $d$ are used as indicated in the caption. The dotted line shows a slope of 0.5 which accounts for the very early stages of growth when $d$ is small.

Let us conclude by briefly summarizing our results: The time development of roughness in quenched disorder depinning models implies a size dependence which has not been noted before. This implies a violation of the scaling relation $\zeta=z \beta$. Instead, we show that the activity is localized even in the early stages of growth and thus this implies a power-law increase of the roughness in time with an exponent $\beta$ given in Eq. (10). This has been confirmed through numerical simulations.

\section{ACKNOWLEDGMENTS}

We acknowledge the hospitality of the International Center of Theoretical Physics in Trieste (It.) where this work was completed.

[1] F. Family and T. Vicsek, "Dynamics of fractal surfaces", (World Sci., Singapore, 1991)

[2] H. Halpin-Healy and Y.C. Zhang, Phys. Rep. 254, 215, (1995)

[3] P. Meakin, "Fractals, Scaling and Growth far from Equilibrium", (Cambridge Univ. Press, Cambridge, 1997)

[4] T. Nattermann, S. Stepanow, L.H. Tang and H. Leschhorn, J. Physique 2, 1483, (1992)

[5] H. Leschhorn, T. Nattermann, S. Stepanow and L.H. Tang, Ann. Phys. ,9, 1 (1997)

[6] O. Narayan and D.S. Fisher, Phys. Rev. B 48, 7030, (1993)

[7] S.V. Buldyrev, A.L. Barabási, F. Caserta, S. Havlin, H.E. Stanley and T. Vicsek, Phys. Rev. A 45, R8313, (1992)

[8] L.H. Tang and H. Leschhorn, Phys. Rev. A 45, R8309, (1992)

[9] J.P. Bouchaud, E. Bouchaud, G. Lapasset and J. Planes, Phys. Rev. Lett. 71, 2240, (1993)

[10] E. Bouchaud, J. Phys. Condens. Matter, 9, 4319 (1997).

[11] J. Schmittbuhl and K.J. Måløy, Phys. Rev. Lett. 78, 3888, (1997).

[12] S. Ramanathan, D. Ertas and D.S. Fischer, Phys. Rev. Lett., 79, 873, (1997).

[13] H. Gao and J.R. Rice, J. Appl. Mech., 56, 828 (1989).

[14] J.F. Joanny and P.G. de Gennes, J. Chem. Phys. 81, 552, (1984) 
[15] A. Paterson, Annales de Physique, 21, 337, (1996).

[16] A.I. Larkin and Y.N. Ovchinnikov, J. Low Temp. Phys. 34, 409, (1979). For a review, see T. Giamarchi and P. Le Doussal, in "Spin Glasses and Random fields", edited by A.P. Young, (World Sci., Singapore,1997).

[17] B.N.J. Persson, "Sliding Friction (Physical Principles and Applications)", (Springer-Verlag, Heidelberg, 1998).

[18] C. Caroli and P. Nozieres, in "The physics of sliding friction", edited by B.N.J. Persson, Vol. 311 of NATO Advanced Study (Kluwer, Dordrecht, 1996).

[19] Note however that extensions of the simple scaling Eq. 1 have been proposed for some experimental data analysis: S. Morel, J. Schmittbuhl, J.M. Lopez and G. Valentin, Phys. Rev. E, 58, 6999 (1998).

[20] J. Schmittbuhl, S. Roux, J.-P. Vilotte, K.J. Måløy, Phys. Rev. Lett. 74, 1787, (1995).

[21] A. Tanguy, M. Gounelle and S. Roux, Phys. Rev. E 58, 1577, (1998).

[22] A. Tanguy, S. Krishnamurthy, P. Abry and S. Roux, preprint (Condmat/9908212).

[23] S. Roux and A. Hansen, J. Physique I 4, 515, (1994).

[24] L. Furuberg, J. Feder, A. Aharony, T. Jossang, Phys. Rev. Lett. 61, 2117, (1998).

[25] M. Paczuski, S. Maslov and P. Bak, Phys. Rev. E 53, 414, (1995). 